\title{
Review
}

\section{The recent advances in Vascular endothelial growth factor and metabolic syndrome in prostate cancer}

\author{
Ya-nan Zhou ${ }^{1}$, Jin-qi Song ${ }^{1}$, Gang-liang $\mathrm{TU}^{1}{ }^{1}$, Hui X $\mathrm{u}^{1}$ : Meng Ding ${ }^{2}$ \\ ${ }^{1}$ Department of Urology, Affiliated Hospital of Chengde Medical University, Chengde, PR China. \\ ${ }^{2}$ Department of Clinical Laboratory, Affiliated Hospital of Chengde Medical University, Chengde, PR China.
}

\begin{abstract}
:
Prostate cancer is one of the most common male malignant tumors in Western countries. In the United States, there are about 170,000 new cases of prostate cancer in 2019 , ranking second only to lung cancer. ${ }^{1,2}$ In recent years, the incidence of prostate cancer in China is gradually increasing occupying the third place among male urogenital malignancies. The metastasis of prostate cancer mainly depends on blood vessels and lymphatic vessels, and the late discovery and poor prognosis of patients are the main reasons for the high fatality rate. ${ }^{2}$ Relevant epidemiological studies on immigration have found that the incidence of PCa in The Asian population in the United States is as high as 77.8 / 10000, which is similar to local residents and significantly higher than that in the native population in Asia. This suggests that, in addition to race and family history, different diet and lifestyle in China and the West may play an important role in the development and progression of PCa. ${ }^{3}$ Epidemiological studies have shown an association between geographic location and prostate cancer risk. In fact, the incidence of prostate cancer in Western men is 15 times higher than in Asian men. This suggests that environmental factors or lifestyle, especially diet and nutrition, may play a key role in the occurrence and development of prostate cancer. ${ }^{4}$ To some extent, metabolic syndrome reflects the diet and lifestyle of patients and is closely related to the occurrence and development of prostate cancer. In addition, Vascular endothelial growth factor (VEGF) as a kind of multi functional cytokine works through specific role in endothelial cells.It can promote endothelial cell proliferation, migration, and increase Vascular permeability. And VEGF can be induced in the body for blood vessel growth, angiogenesis, is closely related to tumorigenesis and metastasis. Numerous recent studies have shown that VEGF is closely related to the occurrence and development of prostate cancer and metabolic syndrome. ${ }^{5}$ In this paper, multiple physiological and pathological mechanisms of VEGF and metabolic syndrome associated with prostate cancer are reviewed.
\end{abstract}

Key words: Vascular endothelial growth factor;Prostate cancer; Metabolic syndrome

\section{Epidemiological characteristics of prostate cancer complicated with metabolic syndrome}

In 1999, the WHO formally named Metabolic Syndrome (MetS) and defined it. Patients with metabolic syndrome should meet the following three or more conditions: ${ }^{1}$ (1) Central obesity: male waist circumference $>102 \mathrm{~cm}$; female waist circumference $>88 \mathrm{~cm}$, body mass index $(\mathrm{BMI}) \geq 25 \mathrm{~kg} / \mathrm{m} 2$. $^{2}$ Serum triglyceride (TG) $>1.7 \mathrm{mmol} / \mathrm{L}^{3} \quad$ High-density lipoprotein cholesterol (HDL-C) : male $\leq 1.04 \mathrm{mmol}$ $/ \mathrm{L}$, female $\leq 1.2 \mathrm{mmol} / \mathrm{L}^{4}$ Blood pressure (BP) $\geq 130 / 85 \mathrm{mmHg} .{ }^{5}$ Fasting blood glucose (FPG) $\geq 6.1 \mathrm{Lmmol} / \mathrm{L}$. Metabolic syndrome is associated with increased risk and progression of almost all cancers, including prostate cancer. ${ }^{6}$ A study found that the onset age of PCa patients with MetS were significantly younger than patients with prostate

Address of Correspondence: Hui Xu, Department of Urology, Affiliated Hospital of ChengDe Medical University, Chengde 067000, China, email: chengdexuhui@163.com 
cancer alone,and PSA levels were significantly higher than those of patients with prostate cancer alone. However, advanced prostate cancer is more common in MetS patients. ${ }^{7}$ Some studies have suggested that a single Mets parameter is not a determinant of prostate cancer risk, and only the combined presence of several parameters can increase the risk of prostate cancer development. ${ }^{6,8}$ Some studies have suggested that every parameter of MetS is related to prostate cancer: HDL-C, Cholesterolemia and obesity are associated with increased prostate cancer prevalence. ${ }^{9}$ Men with a higher BMI have an increased risk of prostate cancer, and the onset age is earlier and the malignancy is higher.10 Meta analysis showed that for every $5 \mathrm{~kg} / \mathrm{m} 2$ increase in $\mathrm{BMI}$ of prostate cancer patients, the mortality rate was increased by $15 \%$. Mets also affects the prognosis of prostate cancer patients. The longitudinal population study showed a 2.66 -fold increased risk of prostate cancer-specific death in obese men with elevated C-peptide levels. Prostate cancer patients with high BMI and high blood sugar have an increased risk of recurrence after treatment. In another study, hypertension and obesity were identified as independent risk factors for recurrence after radical prostate cancer surgery. For every $5 \mathrm{~kg} / \mathrm{m} 2$ increase in $\mathrm{BMI}$, the risk of postoperative recurrence is increased by $21 \%$.

Evidence has shown that parameters of Mets play two roles in the occurrence and development of prostate cancer. One is to reduce the risk of lowgrade prostate cancer, and the other is to promote its development to advanced and invasive prostate cancer. ${ }^{11}$ In one study, patients with Mets were more likely to be aggressive than those with prostate cancer alone. In addition, the waist circumference, fasting glucose, triglycerides, highdensity lipoprotein cholesterol and hypertension of high-grade PCa were significantly higher than those of low-grade PCa. ${ }^{10}$

Some previous studies assessing the association between MetS and PCa have been contradictory. Some people found a positive correlation between metabolic syndrome and $\mathrm{PCa}$, while others showed a negative correlation or no correlation. ${ }^{10}$ This significant difference may be due to a number of confounding factors, such as the size of the study, different definitions of MetS, demographic and anthropometric characteristics, etc. For another example, the testosterone and PSA levels of obese and diabetic patients are lower than those of normal people. Meanwhile, drugs such as metformin and statins taken by patients with diabetes, obesity and hypertension have been proven to reduce the risk of tumor onset and death. ${ }^{12}$ These factors may have contributed to the widely divergent findings.

Epidemiological studies have shown that obese patients have a high incidence of high-grade prostate cancer, a high rate of tumor recurrence, and a high likelihood of progression to castrationresistant prostate cancer (CRPC).At the same time, the study also found that the survival mechanism of many tumor cells depends on insulin. A large number of adipocytes not only lead to increased insulin levels, but also increase ig.F-1, estradiol and leptin levels, while adiponectin and testosterone levels decrease. The combined action of some hormones can promote the proliferation and migration of tumor cells and reduce apoptosis.13 At the same time, hypercholesterolemia can promote tumor progression by providing signaling pathways, proliferation and metastasis matrix for tumor cells. ${ }^{14}$ Some studies have reported that high triglyceride level is an increased risk factor for prostate cancer recurrence after radical resection, but at the same time, some studies have indicated that triglyceride level is negatively correlated with the risk of prostate cancer. ${ }^{13}$

\section{Physiological function and characteristics of VEGF}

VEGF is a pluripotent cytokine that is expressed in many normal tissues of humans and animals. VEGF is secreted by endothelial cells, smooth muscle cells and stromal cells. It can be tested with different methods like Northren blot, PT$P C R$, immunohistochemistry and immunofluore scence method. VEGF can be produced and secreted by epithelial cells and smooth muscle cells, and is often at a higher level in tissues during the growth and development of blood vessels. High expression of VEGF is also seen in tumor, fibroma, delayed allergic reaction, hypoxia and other pathological conditions. VEGF can specifically act on vascular endothelial cells and have a variety of physiological functions. 


\subsection{Regulation of the physiological functions of endothelial cells}

VEGF can stimulate human endothelial cells to produce NO, thus relaxing and regulating blood vessels and vascular tensioning, in response to stimuli such as shear stress and circulatory tension.

\subsection{Increased vascular permeability}

The activity of VEGF is 50,000 times as much as histamine action.It is the most powerful known vascular permeability agent.

\subsection{Angiogenesis}

VEGF can selectively promote the mitosis of endothelial cells in the vascular system, specifically acting on smooth muscle cells, fibroblasts and epithelial cells to promote their proliferation, accelerate the migration of endothelial cells, monocytes and osteoblasts, and effectively establish collateral circulation to improve tissue ischemia.At the same time, VEGF induces angiogenesis by increasing vascular permeability and changing components of extracellular matrix. Studies have shown that the proliferation of endothelial cells at the site of vascular strain in rats is significantly accelerated under the action of VEGF, thus accelerating the healing of vascular endothelial cells.

\section{The relationship between VEGF and metabolic syndrome}

\subsection{VEGF and atherosclerosis in hypertension}

Clinical data and epidemiological studies show that the incidence of atherosclerosis in patients with hypertension is significantly increased. Studies have shown that serum VEGF level in hypertensive patients with carotid plaque is significantly higher than that in hypertensive patients without carotid plaque, and serum VEGF level is positively correlated with carotid intima thickness. Local is chemia and hypoxia in atherosclerotic tissues may be the cause of the elevated VEGF level.Therefore, the increase of serum VEGF becomes one of the predictors of the formation of atherosclerotic plaque in patients with hypertension. ${ }^{15}$ At the same time, studies have found that serum VEGF level in patients with hypertension is positively correlated with systolic blood pressure. ${ }^{15}$ Some scholars believe that the increase of serum VEGF level is to maintain the normal function of vascular endothelial cells in patients with hypertension.It is a compensatory response in the organism to accelerate the repair of damaged endothelial cells; It has also been suggested that VEGF can promote angiogenesis in atherosclerosis. ${ }^{16}$

\subsection{VEGF and diabetes}

Studies have shown that the end products of ischemia, hypoxia and high glycosylation can up-regulate serum VEGF level. The researchers found that serum VEGF level of diabetic rats is significantly higher than that of normal rats. Fasting blood glucose is positively correlated with serum VEGF level, suggesting that high level of VEGF is associated with diabetic hyperglycemia and increased degree of peroxide, and plays a related role in the pathophysiology of diabetic neuropathy. ${ }^{17}$ In addition, VEGF has been found to promote the occurrence of diabetic complications such as diabetic nephropathy and diabetic retinopathy. ${ }^{18} \mathrm{VEGF}$ plays a role in the progression of diabetic nephropathy by changing endothelial cell structure and function and increasing glomerular capillary permeability. Meanwhile, VEGF can promote the formation of new blood vessels by acting on vascular endothelial cells specifically, thus accelerating the progression of diabetic retinopathy.

VEGF can increase the vascular permeability, regulate the physiological function of endothelial cells,promote the growth and formation of endothelial cells and new blood vessels, and play a role in the progression of metabolic syndrome disease. Meanwhile, to a certain extent, VEGF reflects the severity of the disease and the therapeutic effect.

\section{Relationship between VEGF and prostate cancer}

There are a variety of potential growth factors in prostate tissues, and VEGF is the most important pro-angiogenic factor, which is expressed not only in normal prostate tissues but also in prostate cancer tissues. ${ }^{19}$ In an experiment with cats, the expression of VEGF in prostate cancer tissues was significantly decreased after castration. Studies have found that VEGF can regulate the transcription capacity of $\mathrm{Bcl}-2$ mRNA, and then regulate $\mathrm{P} 13 \mathrm{~K} / \mathrm{AKT}, \mathrm{PKC}, \mathrm{mTOR}$, and other factors, 
thereby preventing endothelial cell apoptosis. ${ }^{20}$ Some scholars have found that the high expression of VEGF is correlated with the degree of differentiation, metastasis and TNM stage. The expression of VEGF in clinical staging III+IV was obviously higher than that in I+II period. The high expression rate of VEGF in the prostate cancer group with bone metastasis was significantly higher than that in the group without bone metastasis. Studies have found that VEGF in prostate cancer patients is highly expressed and positively correlated with microvascular density, which is closely correlated with tumor metastasis. ${ }^{21}$ The density of microvessels in patients with metastasis was significantly higher than that in patients without metastasis.One study found that new blood vessels were more likely to be generated in invasive nerve tissue than in uninvasive nerve tissue. Increased blood supply promotes further growth and invasion of tumor cells along nerves, as well as disease progression..$^{22}$ In a study that analyzed 32 patients undergoing radical prostate cancer surgery, they concluded that microvascular density could predict pathological stage.These studies have shown that VEGF can be involved in the occurrence and metastasis of prostate cancer by promoting the generation of microvessels and other complex mechanisms.

\subsection{Diagnostic value of VEGF}

A study found that the higher the Gleason score and clinical stage of prostate cancer patients, the higher the expression of VEGF was, which was positively correlated.However, there was no correlation between VEGF expression and age, smoking, bone metastasis, seminal vesicle invasion and positive surgical margins. ${ }^{23}$ In a study, VEGF levels in 68 patients with prostate cancer were compared before and after radical prostatectomy of prostate cancer, and it was found that VEGF levels in the blood of patients with prostate cancer significantly decreased after radical resection..$^{24}$ In a study of 33 patients with confirmed systemic bone metastasis and 33 patients without bone metastasis, the researchers found that VEGF > $9.52 \mathrm{pmol} / \mathrm{mL}$ had the best diagnostic efficacy in diagnosing the occurrence of bone metastasis of prostate cancer. ${ }^{25}$ Previous studies have shown that the strong positive expression rate of VEGF in prostate cancer is significantly higher than that in $\mathrm{BPH}$, and the strong positive expression rate of VEGF in poorly differentiated prostate cancer is higher than that in moderately differentiated prostate cancer. ${ }^{26}$ The above studies indicate that VEGF has a certain clinical value in the diagnosis of prostate cancer.

\subsection{Prognostic and therapeutic value of VEGF in prostate cancer}

VEGF plays a role in the development and metastasis of prostate cancer by acting on vascular endothelial cells and participating in angiogenesis. Many scholars have found that VEGF and VEGF expression in prostate cancer tissues is closely related to tumor development and metastasis, so they believe that VEGF level can be used as an indicator of whether the tumor has metastasis.The expression of VEGF and its receptor in prostate tumor tissue is directly related to Gleason score and lymph node metastasis. The mechanism is that VEGF-C and VEGF-D stimulate mitosis and proliferation of lymphatic endothelial cells through the receptor VEGFR-3, promoting lymphangiogenesis and thereby promoting lymphatic metastasis of the tumor. Serum VEGF level of patients with prostate bone metastasis was higher than that of patients without prostate bone metastasis, and the difference was statistically significant. ${ }^{25}$ Metastatic PCa was associated with higher VEGF levels compared with localized disease. Anti-VEGF therapy has been the target of multiple clinical trials in the treatment of male patients with CRPR, and has achieved a certain degree of progress. ${ }^{27}$ Some researchers believe that the role of angiogenesis in CRPC may be less than that of other cancers, so the dual targeting effect of androgen signal and VEGF has been explored. ${ }^{28}$ Denisa Baci et. al, found that acetyl-I-carnitine can reduce VEGF and have a protective effect on blood vessels in prostate cancer. ${ }^{29}$ At present, bevacizumab, as an effective anticancer target drug, has shown anti-tumor activity, but it fails to improve the overall survival in the treatment of metastatic castrated prostate cancer, which may be related to the interference effect of VEGF redundancy or androgen on the angiogenic pathway. ${ }^{30}$ These findings suggest that VEGF is closely related to the progression of prostate cancer and provide 
evidence for targeted therapy for prostate cancer.

\section{Conclusion}

To sum up, there are complex pathophysiological mechanisms between metabolic syndrome and prostate cancer, but so far, epidemiological and interventional studies have confirmed that metabolic syndrome plays a role in the occurrence, development and recurrence of prostate cancer.VEGF plays an important role in the pathogenesis and progression of prostate cancer patients with metabolic syndrome. VEGF by acting on vascular endothelial cells, endothelial cells growth, increased vascular permeability, promote the growth of new, small blood vessels, play a role in the occurrence and development of the metabolic syndrome, and the change of VEGF in a certain extent reflects the progress of the metabolic syndrome and the treatment effect. Meanwhile, VEGF plays different roles in the growth, metastasis and recurrence of prostate cancer, which plays a great role in the diagnosis and prognosis of prostate cancer. It is necessary to further study and clarify the action mechanism of VEGF, and actively develop endothelial cell protective agents or antagonists, which will certainly provide help for the future treatment and prognosis of prostate cancer.

\section{Reference}

1. Karzai F H, Madan R A, Dahut W L. Metabolic syndrome in prostate cancer: impact on risk and outcomes. Future Oncol,2016,12(16):1947-1955.

2. Siegel R L, Miller K D, Jemal A. Cancer statistics, 2019. CA Cancer J Clin,2019,69(1):7-34.

3. Mitsuzuka K, Arai Y. Metabolic changes in patients with prostate cancer during androgen deprivation therapy. Int J Urol,2018,25(1):45-53.

4. Quagliariello V, Rossetti S, Cavaliere C, et al. Metabolic syndrome, endocrine disruptors and prostate cancer associations: biochemical and pathophysiological evidences. Oncotarget, 2017,8(18):30606-30616.

5. Mazidi M, Rezaie $P$, Kengne $A$, et al. VEGF, the underlying factor for metabolic syndrome; fact or fiction?. Diabetes Metab Syndr,2017,11 Suppl 1:S61-S64.

6. Uzunlulu M, Telci C O, Oguz A. Association between Metabolic Syndrome and Cancer. Ann Nutr Metab,2016,68(3):173-179.
7. Caliskan S, Kaba S, özsoy E, et al. The effect of metabolic syndrome on prostate cancer final pathology. J Cancer Res Ther,2019,15(Supplement):S47-S50.

8. Gacci M, Russo G I, De Nunzio C, et al. Meta-analysis of metabolic syndrome and prostate cancer. Prostate Cancer Prostatic Dis,2017,20(2):146-155.

9. Yoo S, Oh S, Park J, et al. Effects of metabolic syndrome on the prevalence of prostate cancer: historical cohort study using the national health insurance service database. J Cancer Res Clin Oncol,2019,145(3):775-780.

10. Di Francesco S, Tenaglia R L. Metabolic Syndrome and Aggressive Prostate Cancer at Initial Diagnosis. Horm Metab Res,2017,49(7):507-509.

11. Hammarsten J, Damber J E, Haghsheno M A, et al. A stage-dependent link between metabolic syndrome components and incident prostate cancer. Nat Rev Urol,2018,15(5):321-333.

12. Whitburn J, Edwards C M, Sooriakumaran P. Metformin and Prostate Cancer: a New Role for an Old Drug. Curr Urol Rep,2017,18(6):46.

13. Kiwata J L, Dorff T B, Schroeder E T, et al. A review of clinical effects associated with metabolic syndrome and exercise in prostate cancer patients. Prostate Cancer Prostatic Dis,2016,19(4):323-332.

14. Rezaei M M, Rezaei M M, Ghoreifi A, et al. Metabolic syndrome in patients with prostate cancer undergoing intermittent androgen-deprivation therapy. Can Urol Assoc J,2016,10(9-10):E300-E305.

15. Caletti S, Paini A, Coschignano M A, et al. Management of VEGF-Targeted Therapy-Induced Hypertension. Curr Hypertens Rep,2018,20(8):68.

16. Ghazizadeh H, Rezaei M, Avan A, et al. Association between serum cell adhesion molecules with hsCRP, uric acid and VEGF genetic polymorphisms in subjects with metabolic syndrome. Mol Biol Rep,2020,47(2):867-875.

17. Jerić $M$, Vukojević K, Vuica $A$, et al. Diabetes mellitus influences the expression of NPY and VEGF in neurons of rat trigeminal ganglion. Neuropeptides,2017,62:57-64.

18. Wong $T \mathrm{Y}$, Cheung $\mathrm{C} M$, Larsen $\mathrm{M}$, et al. Diabetic retinopathy. Nat Rev Dis Primers,2016,2:16012.

19. Fryczkowski M, Bułdak R J, Hejmo T, et al. Circulating Levels of Omentin, Leptin, VEGF, and HGF and Their Clinical Relevance with PSA Marker in Prostate Cancer. Dis Markers,2018,2018:3852401.

20. Sun S, Gong F, Liu P, et al. Metformin combined with quercetin synergistically repressed prostate cancer cells via inhibition of VEGF/PI3K/Akt signaling pathway. Gene,2018,664:50-57. 


\section{BPKMCH}

21. Eisermann K, Fraizer G. The Androgen Receptor and VEGF: Mechanisms of Androgen-Regulated Angiogenesis in Prostate Cancer. Cancers (Basel),2017,9(4).

22. Ghinea N, Robin B, Pichon C, et al. Vasa nervorum angiogenesis in prostate cancer with perineural invasion. Prostate,2019,79(6):640-646.

23. Lu Shuai. Expression and significance of EN--2 and VEGF in prostate cancer tissues. 2019.

24. Cui Shaowei, Zhou Jun, Ding Degang. Effect of laparoscopic radical resection of prostate cancer on urine flow mechanics and serum VEGF, IGF-I and PSA levels in patients with early localized prostate cancer Modern medicine and health research (electronic version),2020,4(14).

25. Sun Bin, LIU Juzhen, CAI Tietie, et al. The application of monitoring serum TGF- 1, VEGF and IL-6 levels in the diagnosis of bone metastasis of prostate cancer. World Latest Medical Information Abstracts (Continuous
NEPALESE JOURNAL OF CANCER (NJC)

Electronic Journal),20,20(52).

26. Rivera-Pérez J, Monter-Vera M, Barrientos-Alvarado C, et al. Evaluation of VEGF and PEDF in prostate cancer: A preliminary study in serum and biopsies. Oncol Lett,2018,15(1):1072-1078.

27. Jayson $G$ C, Kerbel R, Ellis $L M$, et al. Antiangiogenic therapy in oncology: current status and future directions. Lancet,2016,388(10043):518-529.

28. Eisermann K, Fraizer G. The Androgen Receptor and VEGF: Mechanisms of Androgen-Regulated Angiogenesis in Prostate Cancer. Cancers (Basel),2017,9(4).

29. Baci D, Bruno A, Cascini C, et al. Acetyl-L-Carnitine downregulates invasion (CXCR4/CXCL12, MMP-9) and angiogenesis (VEGF, CXCL8) pathways in prostate cancer cells: rationale for prevention and interception strategies. J Exp Clin Cancer Res,2019,38(1):464.

30. Cereda V, Formica V, Roselli M. Issues and promises of bevacizumab in prostate cancer treatment. Expert Opin Biol Ther,2018,18(6):707-717. 